\section{Mitgliederversammlungen Arbeitsgemeinschaften der Deutschen Röntgengesellschaft e.V.}

Samstag, 31. Mai 2014 08:00 - 08:45 Uhr AG Diagnostische Radiologie arbeits- und umweltbedingter Erkrankungen (DRauE) 10:45 - 11:45 Uhr AG Herz- und Gefäßdiagnostik

\section{Mitgliederversammlung Deutsche Gesellschaft für Interventionelle Radiologie (DeGIR)Mittwoch, 28. Mai 2014 15:30 - 16:30 Uhr} 12:30 - 13:30 Uhr AG Thoraxdiagnostik

15:45 - 16:45 AG Onkologische Bildgebung

16:30 - 17:30 Uhr

AG Kopf-/Halsdiagnostik

16:45 - 17:45

AG Physik \& Technik (APT)

16:45 - 17:45 Uhr

AG Ultraschall (AGUS)

18:00 - 19:30 Uhr

Chefarzt-Forum
Gastrointestinaldiagnostik

Donnerstag, 29. Mai 2014 12:00 - 13:00 Uhr AG Abdominal-/

Freitag, 30. Mai 2014

12:30 - 13:30 Uhr AG Methodik \&

Forschung (AGMF)

12:15 - 13:15 Uhr

AG Mammadiagnostik

13:30 - 14:30 Uhr

AG Pädiatrische Radiologie

14:00 - 15:00 Uhr AG

Muskuloskeletaldiagnostik

18:15 - 19:15 Uhr AG Uroradiologie

und Urogenitaldiagnostik
Mitgliederversammlung

Vereinigung Medizinisch-Technischer

Berufe in der DRG (VMTB)

Donnerstag, 29. Mai 2014

14:10 - 14:40 Uhr; Saal Bucky

Wir möchten Sie herzlich zu der jährlichen Mitgliederversammlung der Vereinigung der Medizinisch-Technischen Berufe in der DRG (VMTB) einladen. 